\title{
Differences using measured and calculated solar radiation in order to estimate the temperature of the conductor in overhead lines
}

\author{
R. Domingo ${ }^{1}$, A. González ${ }^{2}$, M. Mañana ${ }^{1}$, A. Arroyo ${ }^{1}$, M.A. Cavia ${ }^{1}$ and C. del Olmo ${ }^{2}$ \\ ${ }^{1}$ Department of Electrical and Energy Engineering \\ University of Cantabria \\ Avda.Los Castros s/n, 39005 Santander (Spain) \\ Phone number:+0034 942201378, e-mail: mananam@unican.es \\ ${ }^{2}$ Viesgo Distribución, S.L. \\ C/ Isabel Torres 25, 39011 Santander (Spain) \\ Phone number:+0034 942246000 (ext. 359611), e-mail: antonio.gonzalez@ viesgo.com
}

\begin{abstract}
.
In the last 20 years, installed wind power capacity in North of Spain has grown from 24 to more than 1,650 MW. In this geographical region, wind energy farms are located in places that are far away from the transmission networks so they have to be integrated into distribution networks. Build new overhead lines dedicated for a distribution energy is not the best way to increase the evacuation energy of the wind farms because the cost is quite strong. So, the aim to solve these issues, is by means of an increase in the capacity of existing lines using the dynamic management of the network. This paper is devoted to show the difference of the conductor temperature between the parameter of solar radiation measured by a pyranometer and the use of the theoretical solar radiation which is explained in CIGRE TB601 [1] and IEEE Std. 738-2012 [2] algorithms.
\end{abstract}

\section{Key words}

Conductor temperature, Ampacity, Solar radiation, Pyranometer.

\section{Introduction}

Distribution System Operator (DSO) uses dynamic techniques to manage the distribution network. The data which make possible this management are obtained through the steady state heat balance equation.

$$
P j+P m+P s+P i=P c+P r+P w
$$

where:

$P_{j}$ : Joule heating.

Pm: Solar heating

Ps: Magnetic heating.

$P i$ : Corona heating.

Pc: Convective cooling.

Pr: Radiative cooling.

$P w$ : Evaporative cooling.
The steady state equation (1) can be used to calculate the maximum value of current under the real weather conditions at real-time. One of the most important parameter to be considered is solar radiation. CIGRE TB601 [1] includes global radiation intensity as a combination of the direct solar radiation $I_{B}$, the diffuse sky radiation $I d$ and the albedo $F$.

On the other hand, IEEE Std. 738-2012 [2] considers the solar heat intensity at the Earth's surface as a unique parameter to calculate solar radiation.

The two different approaches to the estimation of the solar radiation are compared in this paper and, at the same time, it is analysed how they affect to the calculation of the temperature of the conductor and the dynamic ampacity. Also, the measurement of the pyranometer is used to make this, so it is obtained five different estimated temperatures of the conductor.

Furthermore, two weather stations are considered, which no are so far one to the other, to see the differences and the data is also compared in several days during the year considering winter and summer.

\section{Methodology}

This study has been carried out in the North-West area of Spain.

The weather stations (WS) had been installed in top of a tower, close to the conductor to be operated in a dynamic way. The altitude of both weather stations are around 500 meters above the sea level.

One of them is placed in the length of the line (WS1) and the other one is placed in a substation (WS2). The weather stations are separated 7.20 kilometres. 


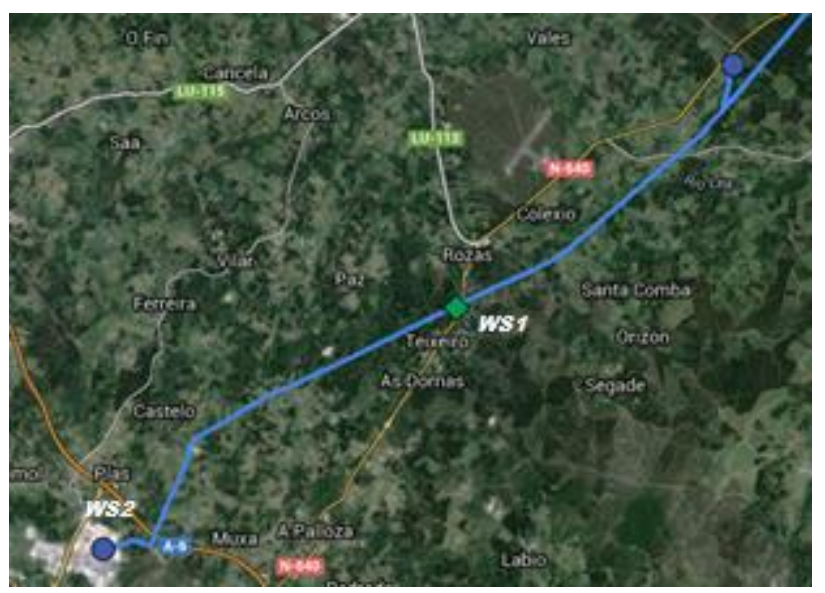

Figure 1. Location of the weather station in an overhead line

Both weather stations are composed of one mechanical anemometer, one thermohygrometer and one pyranometer. Close to the WS1, there is a sensor which is feeded by the current of the line. This sensor provides the measure of conductor temperature and the current of the line.

Solar radiation calculation using CIGRE TB601 [1] and IEEE Std. 738-2012 [2] algorithms provided two different temperatures.

Solar radiation measured by the pyranometer is used in the steady-state equations which are explained in the two algorithms.

At the same time, you can use both the theoretical and the real estimations to calculate four different dynamic ampacity values.

\section{Results}

In this section it is analyzed the results related with the temperature of the conductor and the dynamic ampacity using the weather conditions that has been measured during all the period of time in a winter day and in a summer day by the two weather stations.

Table I shows the meaning of the obtained data and how they have been calculated.

Table I. - Different variables calculated

\begin{tabular}{cc}
\hline VARIABLE & MEANING \\
\hline Pyranometer & $\begin{array}{c}\text { Solar radiation measured by } \\
\text { pyranometer }\end{array}$ \\
SR_CIGRE & $\begin{array}{c}\text { Solar radiation calculated using } \\
\text { CIGRE TB601 }\end{array}$ \\
SR_IEEE & $\begin{array}{c}\text { Solar radiation calculated using IEEE } \\
738\end{array}$ \\
TSMT & $\begin{array}{c}\text { Temperature of the conductor } \\
\text { measured by the sensor on the line } \\
\text { Temperature of the conductor } \\
\text { T_theoretical_CIGRE } \\
\text { calculated using SR_CIGRE as a solar } \\
\text { radiation }\end{array}$ \\
\hline T_theoretical_IEEE & $\begin{array}{c}\text { Temperature of the conductor } \\
\text { calculated using SR_IEEE as a solar } \\
\text { radiation }\end{array}$ \\
\hline
\end{tabular}

Temperature of the conductor calculated using pyranometer value and CIGRE TB601

Temperature of the conductor calculated using pyranometer value and IEEE 738

\section{A. First Comparison: Solar radiation}

Figure $2 \mathrm{a}$, shows the results of solar radiation calculated using the two algorithms and the measurement of the pyranometer in WS1 during a day in winter.

Figure $2 \mathrm{~b}$, shows the results of solar radiation calculated using the two algorithms and the measurement of the pyranometer in WS2 during the same day in winter.

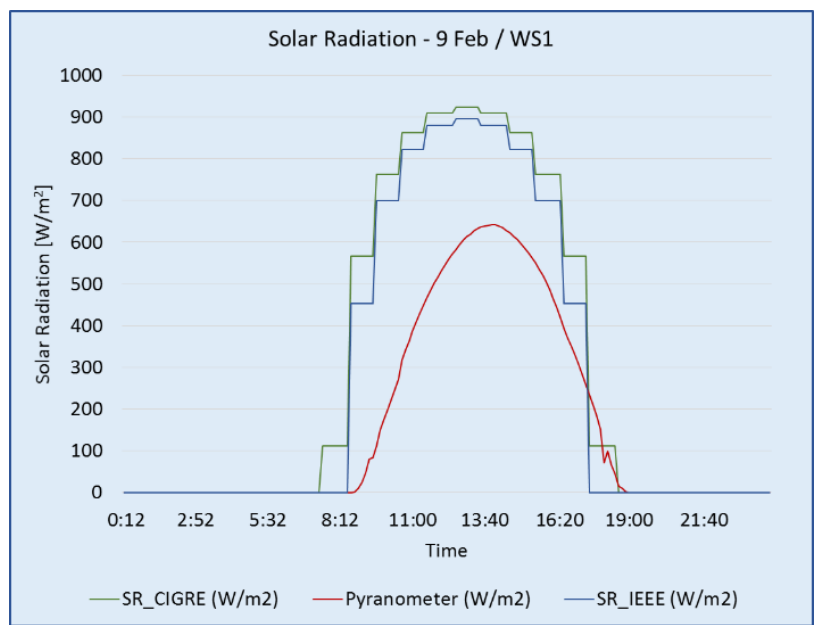

Figure 2a. Different values of solar radiation in a winter day using WS1

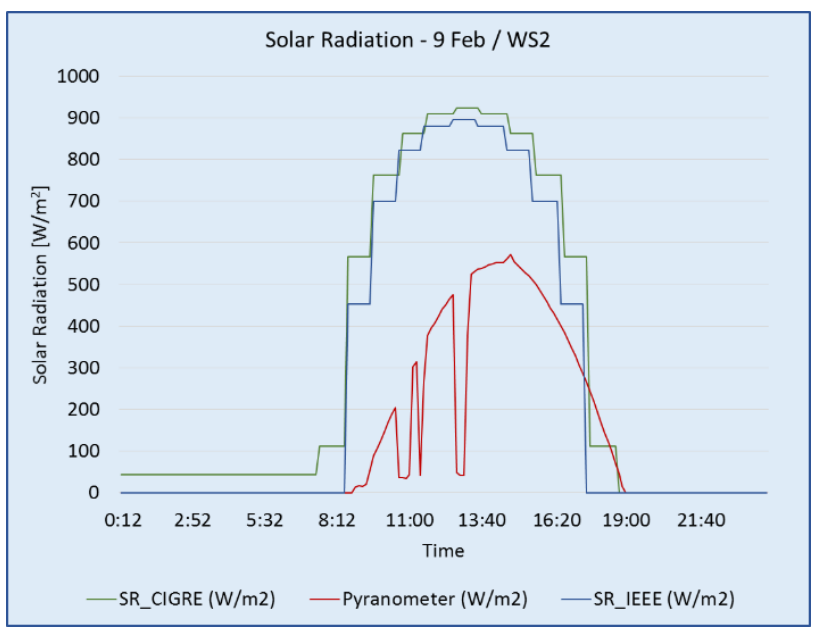

Figure 2b. Different values of solar radiation in a winter day using WS2

Figure 2c, shows the results of solar radiation calculated using the two algorithms and the measurement of the pyranometer in WS1 during a day in summer. 
Figure $2 \mathrm{~d}$, shows the results of solar radiation calculated using the two algorithms and the measurement of the pyranometer in WS2 during the same day in summer.

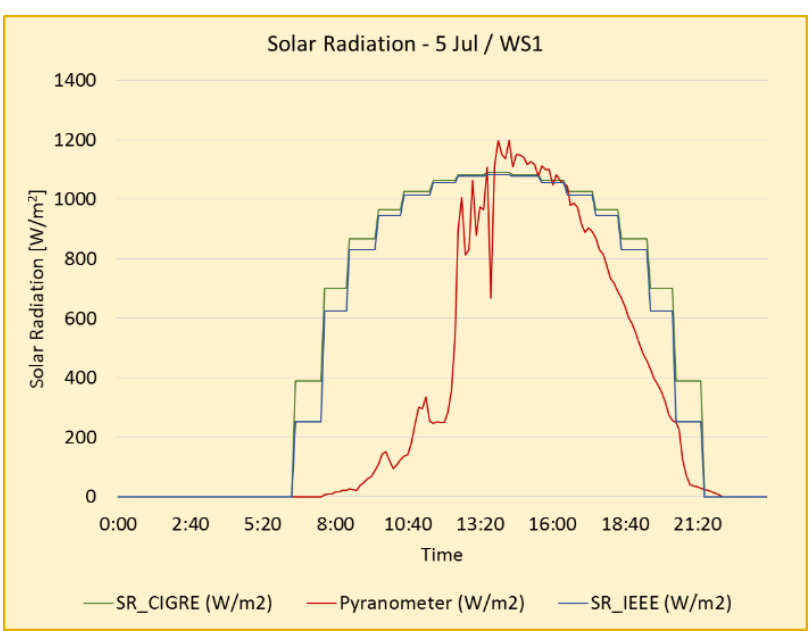

Figure 2c. Different values of solar radiation in a summer day using WS1

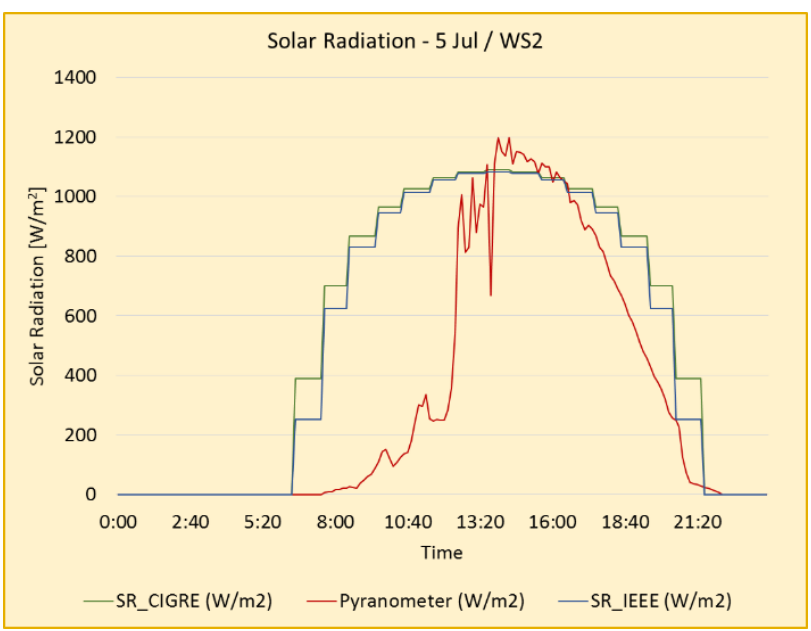

Figure 2d. Different values of solar radiation in a summer day using WS2

If it is performed a comparison between figures $2 \mathrm{a}$ and $2 \mathrm{~b}$. The values of solar radiation using CIGRE TB601 [1] or IEEE Std. 738-2012 [2] are similar but is quite different of the real measurement provided by the pyranometer. Furthermore, it can be seen the effect of the clouds on the pyranometer of the WS2. This is not being considered by both algorithms.

However, if we compare the figures $2 \mathrm{c}$ and $2 \mathrm{~d}$, there are no difference between the use of WS1 or WS2. The difference between the two theoretical values and the real measurement values are quite big. The top of values is similar in the three options, but the real values have a wide hysteresis when the sun rises. The effect when the sun goes down is lower.
On the other hand, if they are compared the figures $2 \mathrm{a}$ and $2 c$ (using the same weather station) it is seen that the theoretical values are more accurate in winter than summer when the sun rises. Nevertheless, when the sun is up theoretical values are more accurate in summer than in winter.

The same happen if they are compared the figures $2 b$ and $2 \mathrm{~d}$.

\section{B. Second Comparison: Temperature of the conductor}

Figure $3 \mathrm{a}$, shows the different temperatures obtained using the two algorithms with two values of solar radiation (measured and calculated) and the real temperature of the conductor that is measured by the sensor in WS1 during a day in winter.

Figure $3 \mathrm{~b}$, shows the different temperatures are obtained using two algorithms with two values of solar radiation (measured and calculated) and the real temperature of the conductor that is measured by the sensor in WS2 during the same day in winter.

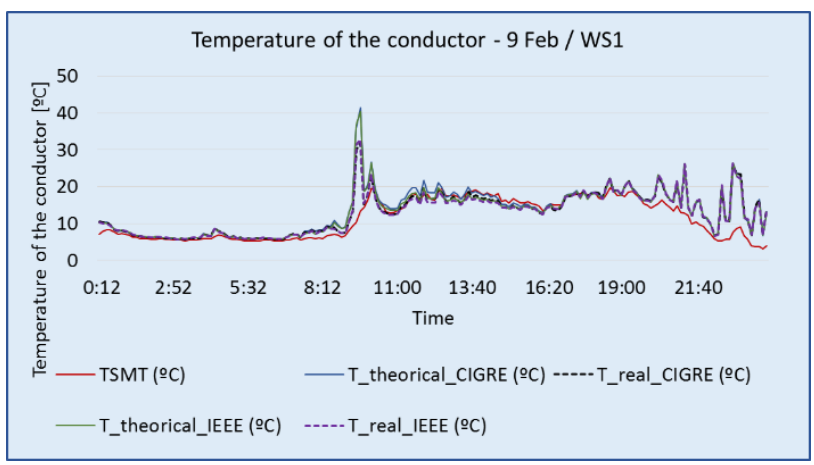

Figure 3a. Different values of the temperature of the conductor in a winter day using WS1

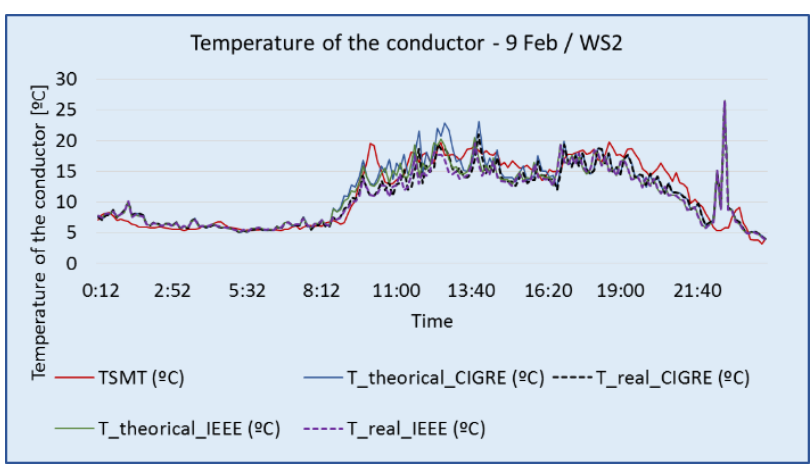

Figure 3b. Different values of the temperature of the conductor in a winter day using WS2

Figure $3 \mathrm{c}$ shows the different temperatures that are obtained using both algorithms with two values of solar radiation (measured and calculated) and the real 
temperature of the conductor that is measured by the sensor in WS1 during a day in summer.

Figure $3 b$, shows the different temperatures obtained using both algorithms with two values of solar radiation (measured and calculated) and the real temperature of the conductor that is measured by the sensor in WS2 during the same day in summer.

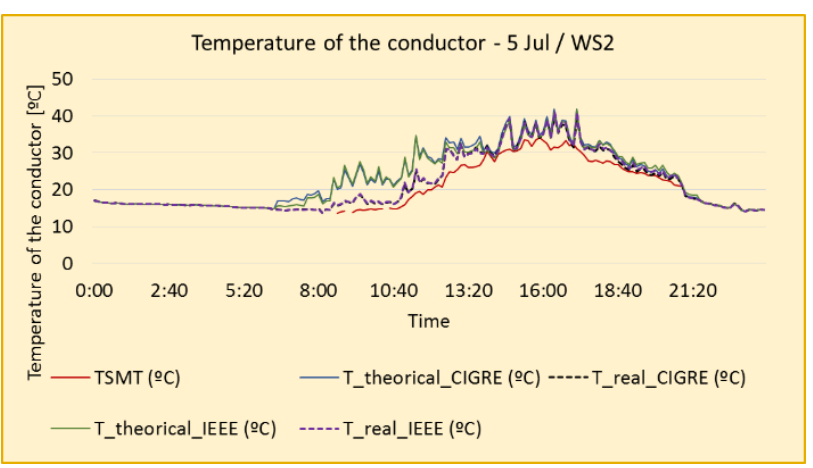

Figure 3c. Different values of the temperature of the conductor in a summer day using WS1

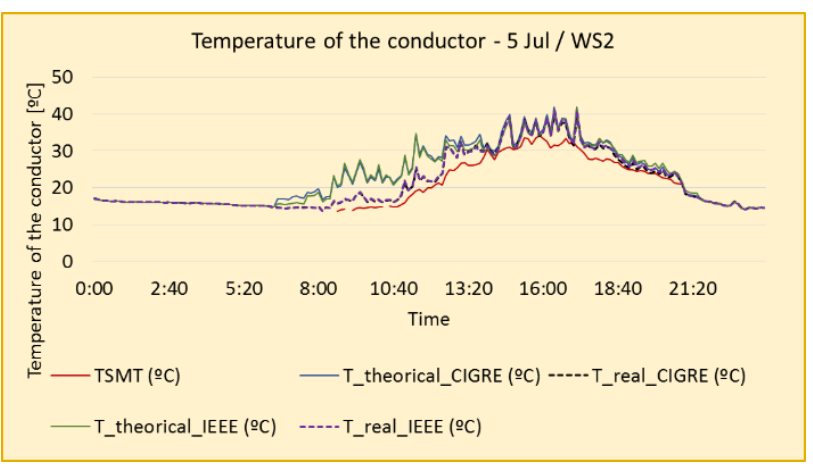

Figure 3d. Different values of the temperature of the conductor in a summer day using WS2

If it is performed a comparison between figures $3 \mathrm{a}$ and $3 \mathrm{~b}$. It is seen that there are no great difference using theoretical or real values of solar radiation in both algorithms to calculate the temperature of the conductor. However there are a difference if they are compared that values with the real temperature of the conductor. In WS1 the difference is the values of both algorithms cannot accurate the real thermal inertia. The same occurs with WS2, but the difference with the real temperature is bigger than using WS1, because the sensor which measure the real temperature of the conductor is placed close WS1 and the distance with the WS2 is about 7.20 kilometers.

The same occurs if we compare figures $3 \mathrm{c}$ and $3 \mathrm{~d}$, but the effect is lower than the situation in a winter day, because the variation of wind speed used to be greater in winter than in summer and the effect of the distance between two weather station is lower.
On the other hand, if we compare the figures $3 \mathrm{a}$ and $3 \mathrm{c}$ (using the same weather station) it is observed that the accuracy is better in winter than in summer. The reason could be that the effect of the wind used to be very important and in summer the wind speed is less than winter and its measurement is less accurate.

The same happen if they are compared the figures $3 \mathrm{~b}$ and $3 d$.

\section{Third Comparison: The error of the temperature of the conductor}

Using the mean squared error to calculate which algorithm is more accurate to estimate the real value of temperature of the conductor. The results shows that CIGRE algorithm is better than IEEE algorithm using real measurement of pyranometer in WS1 (this is WS close the sensor of temperature).

- RMS(TSMT-T_real_CIGRE): 3.9904

- RMS(TSMT-T_real_IEEE): 4,0615

But if the theoretical values are used, IEEE algorithm is better than CIGRE algorithm:

- RMS(TSMT-T_theoretical_CIGRE): 4,5495

- $\quad$ RMS(TSMT-T_theoretical_IEEE): 4,4989

Finally, it is showed that using real values is better than using theoretical values in both algorithms.

In the figure 4 it is showed this errors during a day in winter.

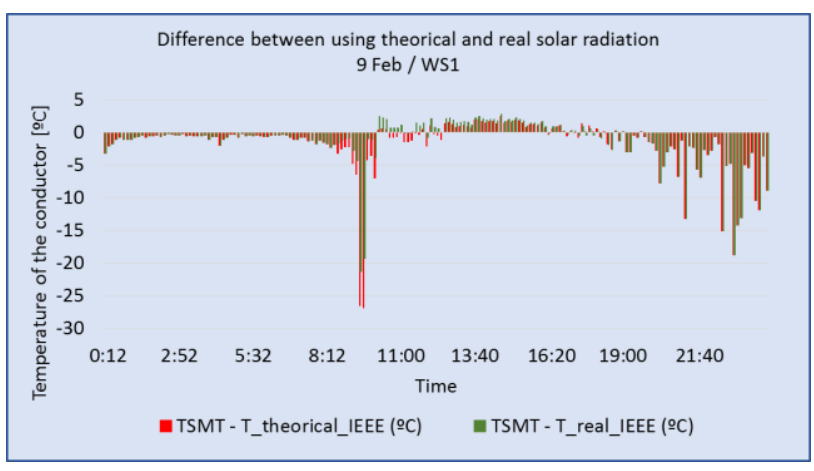

Figure 4. Difference between using theoretical and real values of the solar radiation to calculated the conductor temperature by CIGRE algorithm

\section{Conclusions}

There exists an important difference between real solar radiation measured by a pyranometer and the theoretical solar radiation which are calculated by CIGRE TB601 [1] and IEEE Std. 738-2012 [2]. 
This difference increases when the sun rises and the difference with real temperature measured by a sensor on the line also is important when the sun goes down. The difference reaches in many times $15^{\circ} \mathrm{C}$.

However the difference between using CIGRE TB601 [1] or IEEE Std. 738-2012 [2] are not very important when this values are used to calculate the temperature of the conductor. But there exists a significant difference to use real and theoretical solar radiation to calculate the temperature of the conductor [7].

\section{Acknowledgement}

This work was supported by the Spanish Government under the $\mathrm{R}+\mathrm{D}$ initiative INNPACTO with reference IPT-2011-1447-920000 and Spanish R+D initiative with reference ENE2013-42720-R.

The authors would also like to acknowledge Viesgo for its support.

\section{References}

[1] "Guide for thermal rating calculations of overhead lines”, CIGRE TB601-December 2014.

[2] "IEEE Standard for Calculating the CurrentTemperature of Bare Overhead Conductors", IEEE Std 7382006 (Revision of IEEE Std 738-1993).

[3] A. Madrazo, A. González, R. Martínez, M. Mañana, E. Hervás, A. Arroyo, P.B. Castro, D. Silió. "Increasing Grid Integration of Wind Energy by Using Ampacity Techniques." , in International Conference on Renewable Energies and Power Quality 2013, Bilbao.

[4] H.-J. Dräger, D. Hussels, R. Puffer. "Development and Implementation of a Monitoring-System to Increase the Capacityof Overhead Lines.", E.ONNetz GmbH. CIGRÉ 2008.

[5] "Guide for the selection of weather parameters for bare overhead conductor ratings", CIGRE TB299-2006.

[6] "Guide for Application of Direct Real-Time Monitoring Systems”, CIGRE TB498-2012.

[7] Arroyo, A.; Castro, P.; Martinez, R.; Manana, M.; Madrazo, A.; Lecuna, R.; Gonzalez, A. Comparison between IEEE and CIGRE Thermal Behaviour Standards and Measured Temperature on a 132-kV Overhead Power Line. Energies 2015, 8(12), 13660-13671;

doi:10.3390/en81212391.

http://www.mdpi.com/1996-1073/8/12/12391 